\title{
STEINERT'S DISEASE - HOW THE OUTCOME IS IMPROVED BY REGIONAL ANESTHESIA?
}

\author{
Montenegro L. ${ }^{1}$, Rodrigues A. ${ }^{1}$, Santos H. ${ }^{1}$, Godinho H. ${ }^{2}$ \\ 1- Hospital Garcia de Orta - Almada \\ 2- Centro Hospitalar Trás-os-Montes e Alto Douro
}

\section{BACKGROUND:}

Steinert's disease (SD) is the most common muscular dystrophy in adults. The prevalence of SD affects 1 in 10000 in Europe.

It is a multi-system disorder characterized by skeletal muscle weakness and myotonia, respiratory, cardiac conduction, endocrine, gastrointestinal and other abnormalities.

$\mathrm{SD}$ is a challenge for anesthesiologists due to the exquisite sensitivity to the anesthetic drugs, resulting in risk of a myotonic crisis in the perioperative period and potential postoperative complications (frequency of $8.2 \%$ ). In patients who underwent surgery under general anesthesia, most perioperative complications were related to the pulmonary system.

Whenever possible, general anesthesia should be avoided, favoring regional anesthesia.

This report aims to present a case of ulnar osteosynthesis in a patient with SD and discuss the possible role of regional anesthesia in the outcome of these patients.

\section{METHODS:}

\section{Preoperative}

A 49-year old man, ASA III, with SD was scheduled for ulnar osteosynthesis.

His past medical history was significant for hiatal hernia, obstructive sleep apnea and restrictive pulmonary disease.

His only prior anesthetic exposure was laparoscopic cholecystectomy, with severe respiratory depression requiring intensive care unit admission postoperatively.

There was a full discussion with patient regarding potential risks and complications of general anesthesia. A regional anesthetic technique was proposed, which the patient accepted, after clarifying doubts and concerns.

\section{Intraoperative}

An ultrasound-guided axillary brachial plexus block using $1.5 \%$ mepivacaine $(150 \mathrm{mg})$ and $0.375 \%$ ropivacaine $(37.5 \mathrm{mg})$ was performed.

To reduce the risk of block failure an ultrasound guided technique, in-plane approach and echogenic needles were used.

The operating room was kept warm and a forced-air warming blanket was used to ensure normothermia.

Intraoperatively the patient remained stable and comfortable. No intercurrences were recorded.

\section{Postoperative}

Postoperative course was uneventful with an adequate postoperative pain relief.

The patient was discharged after 2 days.

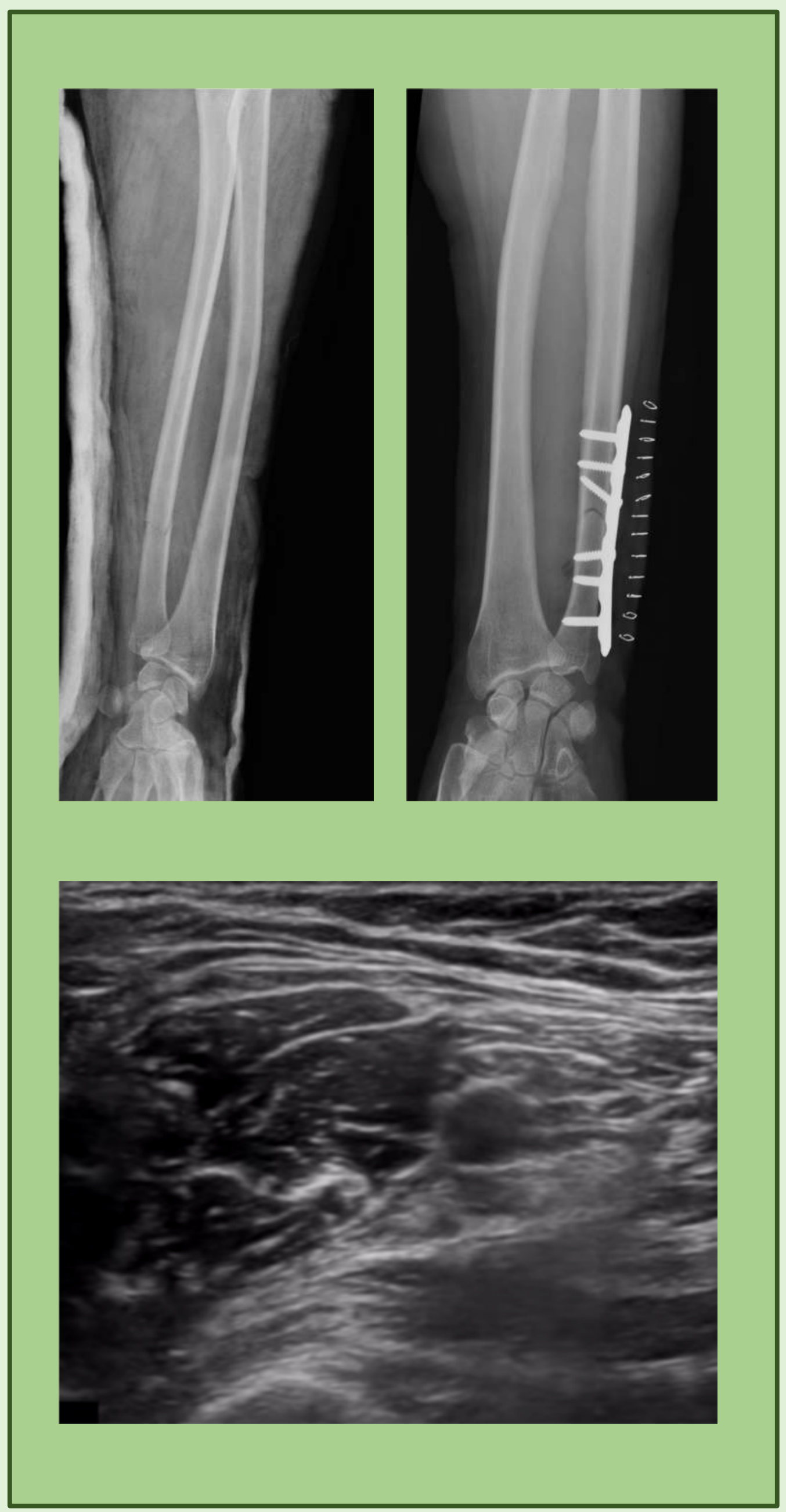

\section{CONCLUSIONS:}

- Meticulous anesthetic management in SD is crucial, as well as the knowledge about anesthetic implications and perioperative complications.

- In this case, regional anesthesia presented a desirable alternative to general anesthesia, effective both in terms of analgesia and ventilatory support avoidance, in the postoperative period.

- In fact, the choice of the adequate anesthetic technique plays a decisive role in the outcome of the patient.

\section{Bibliography}

1. Barash P. G., et al. Clinical Anesthesia. 8th edition. Pgs 614-615, 2017

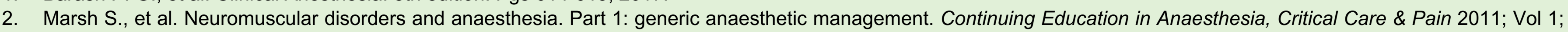
4: $115-118$.

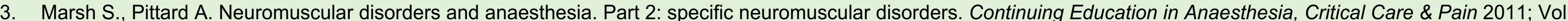
1; 4 : 119-123

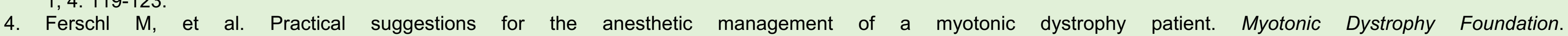
www.myotonic.org/sites/default/files/MDF_LongForm AnesGuidelines_01C.pdf (Accessed on Abril, 2019). 